\title{
Closed Loop Analysis of the Bottleneck Buffer under Adaptive Window Controlled Transfer of HTTP-Like Traffic
}

\author{
Arzad A. Kherani and Anurag Kumar \\ Dept. of Electrical Communication Engg. \\ Indian Institute of Science, Bangalore, 560 012, INDIA \\ email: alam, anurag@ece.iisc.ernet.in
}

\begin{abstract}
We consider an Internet link carrying http-like traffic, i.e., transfer of finite volume files starting at random time instants. These file transfers are controlled by an adaptive window protocol (AWP); an example of such a protocol is TCP.

We provide an analysis for the auto-covariance function of the AWP controlled traffic into the link's buffer; this traffic, in general, is not an on-off process. The analysis establishes that, for Pareto distributed file sizes with infinite second moment, the traffic into the link buffer is long range dependent (LRD).

We also develop an analysis for obtaining the stationary distribution of the link buffer occupancy under an AWP controlled transfer of files sampled from some distribution. The analysis provides a necessary and a sufficient condition for the finiteness of the mean link buffer content; these conditions have explicit dependence on the AWP used and the file size distribution. This establishes the sensitivity of the buffer occupancy process to the file size distribution.

Combining the results from the above analyses, we provide an example in which the closed loop control of an AWP results in finite mean link buffer occupancy even though the file sizes are Pareto distributed (with infinite second moment), and the traffic into the link buffer is long range dependent.

The significance of this work is threefold: (i) it provides a framework for analysing various processes related to the link buffer under AWP controlled transfer of files with a general file size distribution; (ii) it indicates that the buffer behaviour in the Internet may not be as poor as predicted from an open loop analysis of a queue fed with LRD traffic; and (iii) it shows that the buffer behaviour (and hence the throughput performance for finite buffers) is sensitive to the distribution of file sizes.
\end{abstract}

Keywords: long range dependent traffic; models for TCP; processor sharing queue.

\section{INTRODUCTION}

The Internet carries predominantly elastic traffic; the transfer of such traffic is controlled by TCP [1]. Most of the literature on TCP modeling is concerned with the "throughput" obtained by TCP controlled file transfers over a single bottleneck link, with or without the assumption of random drops/losses. This literature can be divided into two streams; the (chronologically) first stream of work assumes a single bottleneck link that is used to transfer a fixed number of files of very large volumes (see [2], [3] and references therein), whereas the second category deals with performance of TCP controlled transfer of http-like (finite, random volume) files where, the number of ongoing transfers is randomly varying (see [4] and its references). An important consideration in the case of http-like traffic is the distribution of file transfer volumes. The file transfer volumes in the Internet has been observed to come from heavy tailed distributions; see [5], for example.

Some of the work that falls in the first category attempts to model the behaviour of the link buffer (see, for example [6], [7]) but, to our knowledge, there is no such analytical study available for TCP controlled transfer of http-like traffic. In this paper we develop a framework for analysing the behaviour of the link buffer, and related processes, assuming that the file transfers are controlled using a general adaptive window protocol (AWP), explicitly taking into account the distribution of file transfer volumes.

We consider the scenario shown in Figure 1 where an Internet link connects clients on one side to servers on the other side. We assume that there is no restriction on the number of simultaneous ongoing transfers. The clients generate file transfer requests and the servers send the requested files using an AWP. The servers and clients are connected to the link by very high speed access links. Hence the Internet link is the bottleneck; also shown in the figure is this link's buffer containing data packets from ongoing file transfers. We make the following system and traffic assumptions:

- The end-to-end propagation delay is negligible (in the sense that the bandwidth delay product is much less than one packet; for example, this could be a 34 Mbps link interconnecting two locations $10 \mathrm{~km}$ apart in a city).

- The link buffer on the server side is such that there is no packet loss. (It follows that since the file sizes are finite, the window growth is governed solely by the increase phase of the AWP; the window of each transfer remains finite since the volume of the transfer is finite. We wish to study the tail behaviour of the stationary contents of the buffer; such an analysis would provide some insight into the tail drop loss behaviour with finite buffers.)

- The link buffer on the server side implements a per flow round-robin scheduling discipline with a service quantum of one packet. Examples of such scheme are Deficit Round Robin (DRR, see [8]) and weighted fair queueing (WFQ).

- Each request is for the transfer of a single file, and the files have independent and identically distributed sizes.

- The starting instants of file transfers constitute a Poisson 
process (of rate $\lambda$ ). (The instants at which new user sessions start is now accepted to be well modelled by a Poisson process (see [9]); our model thus assumes that each session transfers just one file.)

The first assumption above imply that the link buffer contains all unacknowledged data from the ongoing file transfers (sessions).

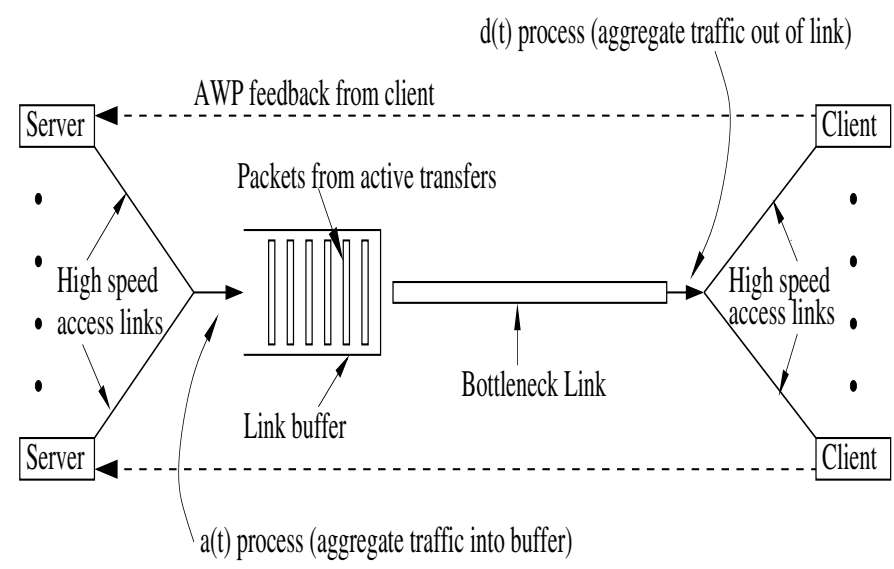

Fig. 1. TCP controlled file transfers over a single link connecting servers and clients; the link propagation delay is assumed to be negligible, and the link buffer is infinite. In this work we analyse the $a(t)$ process, and the link buffer occupancy process.

\section{A. Relationship with Existing Literature}

It has been shown ([10] and [11]) that for Pareto distributed file sizes (with tail $\frac{1}{x^{\alpha}}$ ) the data departure rate process $(d(t)$ in Figure 1) is long range dependent (LRD) with Hurst parameter $\frac{3-\alpha}{2}$. This result follows from the observation that, owing to zero propagation delay, the $d(t)$ process corresponds to the busy idle process of a work conserving queue. Further, $d(t)$ is not affected by the feedback control used. Clearly, however, the input process to the link buffer depends on the feedback control used and hence it is interesting to study the correlation structure of the data arrival rate process into the link buffer (denoted by $a(t)$ in Figure 1); this is one part of the work presented here.

Extensive analysis of Internet data has confirmed that Internet traffic is LRD (see [12]). It has been argued that the LRD behaviour of Internet traffic is related to heavy tailed file transfer volumes ([5]). Recent studies (see [10], [13], [14]) show that the stationary distribution of a queue fed with LRD traffic will have a non-exponential tail; for example, it has been shown that an arrival rate process auto-covariance that is $O\left(\frac{1}{\tau^{\alpha-1}}\right), 1<\alpha<2$, leads to a stationary distribution of buffer occupancy that has a tail that is $O\left(\frac{1}{x^{\alpha-1}}\right)$. The above observations are usually combined to conclude that the link buffer occupancies in the Internet will be heavy tailed. Such observations are, however, based on an "open loop" analysis of an LRD traffic source feeding a buffer. Recent numerical studies ([15], [16], [9]) suggest that an understanding of traffic and buffer processes in the Internet should take into account the closed loop nature of Internet congestion control, namely TCP which is an adaptive window protocol (AWP). In this paper we carry out such an analysis for the network scenario of Figure 1 and for a general AWP.

It is easy to see that the behaviour of a buffer for a given input process can be strikingly different in a feedback loop as compared to when the same process is applied to the buffer (i.e., "open loop"). In Figure 2 we provide one such example. The (a) part of the figure depicts a closed queueing system where a single customer is fed back to the queue (with a new service requirement distributed as exponential $(\mu)$ ) as soon as it gets served; the system is clearly stable as there is always a single customer in the system. Note that the customer arrival instants to the queue form a Poisson process of rate $\mu$. The (b) part of figure depicts an $\mathrm{M} / \mathrm{M} / 1$ queue with a Poisson arrival process of rate $\mu$ and the mean service requirement $\frac{1}{\mu}$; this queue is clearly unstable.

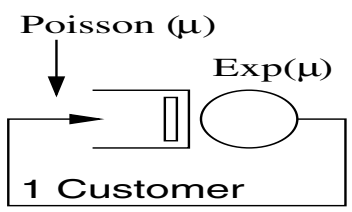

(a)



(b)
Fig. 2. Two simple queueing models illustrating that for the same input process (Poisson with rate $\mu$ ) the behaviour of a buffer is entirely different in a closed loop (a) and in an open loop (b). The queue in (b) is unstable (a null recurrent Markov chain).

\section{B. Overview and Organisation of the Paper}

Assuming an AWP and a general file size distribution, we study the auto-covariance function of the data arrival rate process into the link buffer (the $a(t)$ process, see Figure 1). We then analyse the link buffer occupancy process for a general AWP and file size distribution and provide a necessary and a sufficient condition for the existence of the mean buffer occupancy. Combining the results from above two analysis it is shown that it is possible to have a finite mean link buffer occupancy even when the file size requirements are heavy tailed and the $a(t)$ process is $\mathrm{LRD}^{1}$. This does not contradict the result of [10], [13] as the model analysed there does not include any feedback control from the queue.

The paper is organised as follows. In Section II we develop a queueing equivalent model of the scenario of Figure 1, introduce some notation we use in the paper and give some queueing results required later in the work. In Section III we introduce some characterising functions associated with an AWP. Section IV presents a study of the auto-covariance function of the $a(t)$ process. In Section $\mathrm{V}$ we give the analysis of the link buffer occupancy process.

\section{MODEling APPROACH}

The contents of the link buffer comprise the windows of each of the active flows; since the propagation delay is zero, the entire window of an active flow is in the link buffer. These windows are served in a round-robin manner as per

\footnotetext{
${ }^{1}$ Our recent work gives the exact tail behaviour of the buffer occupancy distribution, see [17].
} 
the assumption of per flow fair scheduling at the link buffer. The round-robin discipline is simpler to study via the PS model hence we approximate the service of the windows in the link buffer by a PS discipline. Based on this approximation, Figure 3 depicts the queueing equivalent of the scenario shown in Figure 1; note that the link buffer has been replaced by a PS server. Owing to zero link propagation delay, each active flow has positive amount of outstanding data (window) in the link buffer and since these windows are served in a PS fashion, it follows that the ongoing file transfers (as a whole) also get service in a PS manner ${ }^{2}$.

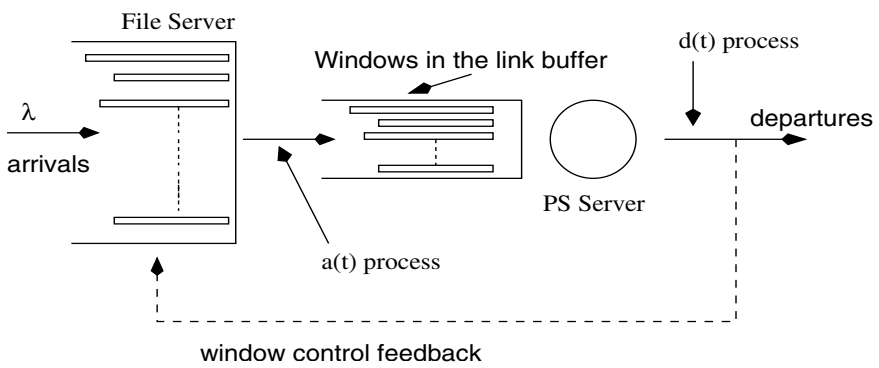

Fig. 3. Queueing equivalent of the network scenario of Figure 1.

Note that at any time instant $t$, an active session would have successfully transferred some data to its client, some of its data would be in the link buffer (this would be the current window size of TCP controlling the transfer of the file), and the remaining data would be in the server waiting to be transferred. At any time instant we use the term "age" for the amount of data of a file successfully transferred to the client, and by "residual file" or "residual service" we mean the amount of data of the file yet to be transferred, i.e., the sum of the data in the link buffer and that remaining in the server. As data from a file is served (along with other files, in a PS manner) more data is pulled into the link buffer from the file server, so as to replenish the windows and to account for any window growth. Eventually the amount of data of the file in the server reduces to zero, and the entire residual file is in the link buffer. Note that as long as the file is not fully transferred, a positive amount of it is always in the link buffer. Thus in term of the PS queue model, the server is the link, and each "customer" in service is split between the file server and the link buffer.

The above general model, which was motivated by observations about the performance of TCP controlled finite volume transfers, is what we work with in this paper. We will show how to apply it to specific cases of TCP type adaptive window control.

\section{A. Notation and Some Results Related to an M/G/1 PS Queue}

We follow the convention that if $Z$ is a random variable then

\footnotetext{
${ }^{2}$ Recent literature [18], [4] suggests that, for a zero propagation delay link and even in absence of a per flow fair scheduling at the link buffer, in the above situation the TCP mechanism effectively serves the files in a Processor Sharing (PS) fashion. This suggests that, even if the packets are served in the order of their arrival to the link buffer, the packets from the active files are interlaced in such a manner that the data from these files in the link buffer is served in a round robin manner. We have observed, however, that such an equal sharing is not valid for general file size distributions and breaks down, in particular, for heavy tailed file volume distributions unless the link buffer implements a per flow fair queueing.
}

$E Z$ is its expectation, $Z(\cdot)$ denotes its cumulative distribution function (cdf), $Z^{c}(\cdot)$ its complementary cdf, $z(\cdot)$ its density (if it exists), $\tilde{z}(s)$ the Laplace Stieltjes Transform (LST) of $Z(\cdot)$, and $\tilde{Z}(s)$ the Laplace Transform (LT) of $Z(\cdot)$. We also let $Z_{e}(\cdot)$ denote the excess distribution of $Z(\cdot)$, and $Z_{s}(\cdot)$ denotes the spread distribution associated with $Z$ (see [19]).

In the context of a queueing system, with the above mentioned convention, we introduce the following notation

$V$ the random variable for the file sizes brought by sessions,

$\rho \quad:=\lambda E V$,

$a(t)$ the instantaneous data arrival rate into the queue at time $t$,

$N(t)$ number of sessions active at time $t$,

$Y(t)$ total of the residual file sizes at time $t$,

$B$ the busy period random variable of an M/G/1 queue,

$x(s) \quad:=1-\tilde{b}(s),($ introduced for notational convenience),

$B_{y} \quad$ the busy period random variable with initial 'work' $y$ in an $\mathrm{M} / \mathrm{G} / 1$ queue,

$K_{y}(t)$ the number of starts of idle periods in an $\mathrm{M} / \mathrm{G} / 1$ queue until time $t$ given $Y(0)=y$,

We take the link speed to be unity, hence $\rho$ is also the normalised offered load on the link.

We know that, (see [19]),

$$
\begin{gathered}
\tilde{b}_{y}(s)=e^{-y(s+\lambda-\lambda \tilde{b}(s))}=e^{-y(s+\lambda x(s))} \\
\tilde{b}(s)=\tilde{v}(s+\lambda x(s))
\end{gathered}
$$

We use the notation $f(t) \sim_{t \rightarrow t_{0}} g(t)$ to mean $\lim _{t \rightarrow t_{0}} \frac{f(t)}{g(t)}=1$ and write $f(t) \succeq_{t \rightarrow t_{0}} g(t)$ to mean that there exists a function $h(t)$ such that $f(t) \geq h(t)$ for all $t$ and $h(t) \sim_{t \rightarrow t_{0}} g(t)$.

In this work we frequently use the following known results for a stationary M/G/1 PS queue (see [20]). At any time instant $t$

- $P\{N(t)=n\}=(1-\rho) \rho^{n}$

- The total file sizes of each of the $N(t)$ ongoing transfers at time $t$ are mutually independent random variables and are distributed as $V_{s}(\cdot), v_{s}(x)=\frac{x v(x)}{E V}$, (see [19]),

- Conditioned on the total service requirement of a file transfer being $v$, its age, (i.e., the data already transferred to the client by the session by time $t$ ) is uniformly distributed over the interval $[0, v]$.

A recent work ([21]) reports the following result.

- In the M/G/1 PS system modified by having $k$ permanent jobs with infinite service requirements, for $n \geq k$,

$$
\lim _{t \rightarrow \infty} P\{N(t)=n\}=(1-\rho)^{k+1}\left(\begin{array}{c}
n-k \\
k
\end{array}\right) \rho^{n}
$$

\section{Characterisation of a General AWP}

An AWP can be characterised by the amount of data released by the sender (server) in response to a unit amount of acknowledged data. In general, this quantity will be a function of the size of the file being transferred and the total amount of data successfully received by the client. We introduce the following notation in the context of a general 
AWP.

\section{Notation}

$R_{v}(u)$ amount of data released by the sender per unit of acknowledged data when a file of size $v$ has attained age $u$ (i.e., $u$ amount of its data has been acknowledged),

$U(v)=\sup \left\{u: R_{v}(u)>0,0 \leq u \leq v\right\} \quad$ is the age of a file (of size $v$ ) after which an acknowledgment does not result in release of data from file server, i.e., exactly at this age the file server has sent a total of $v$ amount of data to the link buffer. At this point, the receiver would have acknowledged $U(v)$ amount of data and $v-U(v)$ amount of data from the file will be in the link buffer,

$X(u)=\inf \{v: U(v)>u, 0 \leq v<\infty\}$ is the minimum file size for which the protocol will still be sending data to the buffer after it has received acknowledgment of $u$ amount of data

Note that,

$$
\begin{aligned}
\int_{u=0}^{U(v)} R_{v}(u) d u & =v \\
U(X(u)) & =u \\
X(U(v)) & =v
\end{aligned}
$$

Thus, $U$ and $X$ are inverse functions.

In the following we obtain the $R_{v}(u), U(v)$, and $X(u)$ functions for the AWPs that we study later in this paper.

\section{A. The $R_{v}(u), U(v)$ and $X(u)$ Functions for TCP's Slow Start}

If the transfer of a file is controlled using only the slow start algorithm of TCP then each unit of data acknowledged results in the arrival of two units of data, thus

$$
R_{v}(u)=2 I_{\{u \leq U(v)\}}
$$

Using Equation 4 with $R_{v}(u)=2 I_{\{u \leq U(v)\}}$, we get

$$
U(v)=\frac{v}{2}
$$

Using Equation 5 with $U(v)=\frac{v}{2}$, we get

$$
X(u)=2 v
$$

\section{B. The $R_{v}(u), U(v)$ and $X(u)$ Functions for TCP's Conges-} tion Avoidance

If the transfer of a file is controlled using only the congestion avoidance algorithm of TCP then, when window size is $w$, each unit of data acknowledged results in arrival of $1+\frac{1}{w}$ units of data. Also, when the window size is some integer $n$, the amount of data that has been acknowledged (i.e., the age of file) is $\frac{n(n-1)}{2}$. Thus, the window size corresponding to an age of $u$ is $\frac{1+\sqrt{1+8 u}}{2}$. To avoid complex expressions, since we are interested in asymptotic behaviour, we use the approximation that when the age of file is $u$ the window size is $\sqrt{2 u}$. This gives

$$
R_{v}(u)=\left(1+\frac{1}{\sqrt{2 u}}\right) I_{\{u \leq U(v)\}}
$$

Using Equation 4 with $R_{v}(u)=\left(1+\frac{1}{\sqrt{2 u}}\right) I_{\{u \leq U(v)\}}$, we get

$$
U(v)=v+1-\sqrt{1+2 v}
$$

Using Equation 5 with $U(v)=v+1-\sqrt{1+2 v}$, we get

$$
X(u)=u+\sqrt{2 u}
$$

\section{ASYmptotic Behaviour of the Auto-COVARIANCE FUNCTION OF THE $a(t)$ PROCESS}

In this section we study the auto-covariance function of the $a(t)$ process for the stationary system (see Figure 3 ) when the transfer of files is controlled by an AWP.

When there are $n$ active sessions, owing to the PS model, a unit data served by the link implies each of these $n$ sessions gets a service of $\frac{1}{n}$ data units hence (recalling that the link speed is unity) the total data sending rate at instant $t$ will be

$$
a(t)=\sum_{i=1}^{N(t)} \frac{R_{v_{i}}\left(u_{i}(t)\right)}{N(t)}
$$

where $u_{i}(t)$ and $v_{i}$ are, respectively, the total service received by and the total service requirement of the $i^{t h}$ session active at time $t$.

Lemma 4.1: For the stationary system,

$$
E a(t)=\rho
$$

Proof: By conditioning on $N(t)$ and the file size requirements $\left(v_{i}\right)$ of the $N(t)$ ongoing transfers and their ages $\left(u_{i}\right)$, using Equation 6 and results of Section II-A,

$$
\begin{aligned}
E a(t)= & \sum_{n=1}^{\infty}(1-\rho) \rho^{n} \int_{v_{1}=0}^{\infty} \ldots \int_{v_{n}=0}^{\infty} \int_{u_{1}=0}^{v_{1}} \ldots \int_{u_{n}=0}^{v_{n}} \\
& {\left[\sum_{i=1}^{n} \frac{R_{v_{i}}\left(u_{i}\right)}{n}\right] \frac{d u_{n}}{v_{n}} \ldots \frac{d u_{1}}{v_{1}} d V_{s}\left(v_{n}\right) \ldots d V_{s}\left(v_{1}\right) } \\
= & \sum_{n=1}^{\infty}(1-\rho) \rho^{n} \int_{v_{1}=0}^{\infty} \int_{u_{1}=0}^{v_{1}} R_{v_{1}}\left(u_{1}\right) d u_{1} \frac{d V_{s}\left(v_{1}\right)}{v_{1}}
\end{aligned}
$$

Using Equation 4,

$$
\begin{aligned}
E a(t) & =\sum_{n=1}^{\infty}(1-\rho) \rho^{n} \int_{v_{1}=0}^{\infty} v_{1} \frac{d V_{s}\left(v_{1}\right)}{v_{1}} \\
& =\rho
\end{aligned}
$$

We note that this result is as would be expected; for let $A(t)$ denote the cumulative process for the rate process $a(t)$ (i.e., $\left.A(t)=\int_{u=0}^{t} a(u) d u\right)$. Then for the stable system, the rate of data leaving the server should be equal to the rate of data requested by the clients, i.e.,

$$
\lim _{t \rightarrow \infty} \frac{A(t)}{t}=\lambda E V=\rho
$$


The auto-covariance function of the $a(t)$ process for a lag of $\tau$ is given by

$$
\begin{aligned}
r_{a}(\tau):= & E a(0) a(\tau)-E a(0) E a(t) \\
= & E a(0) a(\tau)-\rho^{2} \\
= & E a(0) a(\tau) I_{\left\{N(0)>0, K_{Y(0)}(\tau)=0\right\}} \\
& +E a(0) a(\tau) I_{\left\{N(0)>0, K_{Y(0)}(\tau)>0\right\}}-\rho^{2} \\
= & J_{1}(\tau)+J_{2}(\tau)-\rho^{2}
\end{aligned}
$$

where $K_{y}(\cdot)$ and $Y(t)$ are as defined in Section II-A. We study the asymptotic behaviour of $r_{a}(\tau)$ by considering $J_{1}(\tau)$ and $J_{2}(\tau)$ separately.

Theorem 4.1: For AWP controlled transfer of file sizes of distribution $V(\cdot)$, if there exists an $r$ such that $0<r \leq$ $\inf _{0 \leq v<\infty} \inf _{0 \leq u<U(v)} R_{v}(u)$, then

$$
J_{1}(\tau) \succeq_{\tau \rightarrow \infty}(1-\rho)^{2} \lambda r^{2} \int_{v=X(\tau)}^{\infty} \int_{u=0}^{U(v)-\tau} d u d V(v)
$$

Proof: See Appendix I.

As is evident from the examples of slow start and congestion avoidance given in Section III, the $R_{v}(u)$ function for an AWP, in general, will be of the form $R(u) I_{\{0 \leq u \leq U(v)\}}$ so that $\inf _{0 \leq v<\infty} \inf _{0 \leq u<U(v)} R_{v}(u)=\inf _{u \geq 0} R(u)$. Here $R(u)$ can be thought of as the data sending rate function of a file of infinite volume.

Pareto distributed file sizes have the following distribution,

$$
V^{c}(x)=\min \left(1, \frac{1}{x^{\alpha}}\right)
$$

with the property that $E V$ is finite iff $\alpha>1$ and $E V^{2}$ is finite iff $\alpha>2$.

Corollary 4.1: For Pareto distributed file sizes transferred using only the TCP's slow start phase,

$$
J_{1}(\tau) \succeq_{\tau \rightarrow \infty} 4(1-\rho)^{2} \frac{\lambda}{\alpha-1} \frac{1}{2^{\alpha} \tau^{\alpha-1}}
$$

Proof: For slow start phase of TCP, $R_{v}(u)=$ $2 I_{\{u<U(v)\}}, U(v)=\frac{v}{2}$ and $X(\tau)=2 \tau$. Use Theorem 4.1 with $r=2$.

Corollary 4.2: For Pareto distributed file sizes transferred using only the TCP's Congestion avoidance phase,

$$
J_{1}(\tau) \succeq_{\tau \rightarrow \infty}(1-\rho)^{2} \frac{\lambda}{\alpha-1} \frac{1}{\tau^{\alpha-1}}
$$

Proof: For congestion avoidance phase of TCP, $R_{v}(u)=(1+$ $\left.\frac{1}{\sqrt{2 u}}\right) I_{\{u<U(v)\}}, \quad U(v)=v+1-\sqrt{1+2 v}$ and $X(u)=u+$ $\sqrt{2 u}$. Now,

$$
\begin{aligned}
& U(v)=(v+1-\sqrt{1+2 v})=\frac{(\sqrt{1+2 v}-1)^{2}}{2} \\
& >\frac{(\sqrt{2 v}-1)^{2}}{2}=\frac{2 v+1-2 \sqrt{2 v}}{2}>v-\sqrt{2 v}
\end{aligned}
$$

Hence, from Theorem 4.1 with $r=1$,

$$
\begin{gathered}
J_{1}(\tau) \succeq_{\tau \rightarrow \infty}(1-\rho)^{2} \lambda\left[\int_{v=\tau+\sqrt{2 \tau}}^{\infty}(v-\sqrt{2 v}) d V(v)\right. \\
\left.-\tau V^{c}(\tau+\sqrt{2 \tau})\right]
\end{gathered}
$$

$$
\begin{aligned}
=(1-\rho)^{2} \lambda\left[\frac{E V}{(\tau+\sqrt{2 \tau})^{\alpha-1}}-\frac{\frac{\alpha}{(\alpha-0.5)}}{(\tau+\sqrt{2 \tau})^{\alpha-0.5}}\right. & \left.-\frac{\tau}{(\tau+\sqrt{2 \tau})^{\alpha}}\right] \\
\sim_{\tau \rightarrow \infty}(1-\rho)^{2} \frac{\lambda}{\alpha-1} \frac{1}{\tau^{\alpha-1}} &
\end{aligned}
$$

The proof follows by noting that $f(t) \succeq_{t \rightarrow \infty} g(t)$ and $g(t) \sim_{t \rightarrow \infty} h(t)$ implies $f(t) \succeq_{\tau \rightarrow \infty} h(t)$.

Theorem 4.2: For AWP controlled transfer of Pareto distributed file sizes,

$$
\begin{aligned}
\rho^{2}-J_{2}(\tau) & \rightleftharpoons \quad \frac{\rho^{2}}{s}-\tilde{J}_{2}(s) \\
& \sim_{s \rightarrow 0} \rho \lambda \frac{\alpha^{2} \Gamma(-\alpha)}{(1-\rho)^{\alpha-1} s^{2-\alpha}}
\end{aligned}
$$

Proof: See Appendix II.

Corollary 4.3: For AWP controlled transfer of Pareto distributed file sizes with $\alpha>1$,

$$
\rho^{2}-J_{2}(\tau) \sim_{\tau \rightarrow \infty} \frac{\rho^{2}}{(1-\rho)^{\alpha-1}} \frac{1}{\tau^{\alpha-1}}
$$

Proof: Follows using Theorem 4.2 and a Tauberian theorem from [11] (reproduced, for convenience, in Appendix III).

Note that the asymptotic behaviour of $\rho^{2}-J_{2}(\tau)$ is independent of the AWP used to transfer the files. A relation between the above asymptotic behaviour and the convergence rate in the Key Renewal Theorem (see [19]) can also be easily seen.

Theorem 4.3: For transfer of Pareto distributed file sizes under slow start or congestion avoidance phase of TCP,

$$
r_{a}(\tau) \succeq_{\tau \rightarrow \infty} \Delta(\lambda, \alpha) \frac{1}{\tau^{\alpha-1}}
$$

For some function $\Delta(\lambda, \alpha)$ that depends on whether slow start or congestion avoidance is used to transfer the files.

Further, there exists a $\lambda^{*}>0$ such that $\Delta(\lambda, \alpha)>0$ for all $0<\lambda<\lambda^{*}$

Proof: Using Corollaries 4.1, 4.2 and 4.3,

$$
\begin{aligned}
& r_{a}(\tau)=J_{1}(\tau)+J_{2}(\tau)-\rho^{2} \\
& \succeq_{\tau \rightarrow \infty}\left[\delta(1-\rho)^{2}-\frac{\lambda \alpha^{2}}{(\alpha-1)(1-\rho)^{\alpha-1}}\right] \frac{\lambda}{(\alpha-1) \tau^{\alpha-1}} \\
&=\Delta(\lambda, \alpha) \frac{1}{\tau^{\alpha-1}}
\end{aligned}
$$

where $\delta=1$ for congestion avoidance and $\delta=2^{2-\alpha}$ for slow start. Note that in both cases $\delta$ is independent of $\lambda$. Now, for fixed $\alpha>1$ and $\rho<1$, the second term in square brackets above is always positive and decreases to 0 as $\lambda \rightarrow 0$ while the first term increases to $\delta$ (a positive number) as $\lambda \rightarrow 0$. Since both these terms are continuous functions of $\lambda$, there exists $\lambda^{*}>0$ such that $\frac{\Delta(\lambda, \alpha)}{\lambda}>0$ for all $\lambda<\lambda^{*} . \bullet$

It follows from Theorem 4.3 that, for $1<\alpha<2$ and $\lambda<\lambda^{*}$, the process a $(t)$ is LRD. Further, if it can be shown that $r_{a}(\tau)$ has a hyperbolic decay then the a $(t)$ process will be LRD with Hurst parameter $H \geq \frac{3-\alpha}{2}$. 


\section{Analysis of the Stationary Link Buffer Process}

In this section we present the analysis for obtaining the distribution of the link buffer content process. An explicit expression for the Laplace transform of the buffer content process is obtained in terms of the file size distribution and the quantities associated with an AWP (see Equations 8 and 10). The structure of the Laplace transform thus obtained is used to find a necessary and a sufficient condition for existence of the mean of buffer content process.

\section{Important Observations}

- The amount of data in the link buffer at any time $t$ is the sum of the windows from all the file transfers ongoing at $t$.

- Owing to the no packet loss assumption, and AWP follows a known window increase schedule. This enables us to determine the window (which is also the session's contribution to the link buffer occupancy) for a given age.

The age of an ongoing transfer can be obtained in the following way: the distribution of the stationary number of ongoing transfers, $N$, is given by $P\{N=n\}=(1-\rho) \rho^{n}$. Conditioned on the number of ongoing transfers, the ages of the various ongoing transfers are independent; further, the age of an ongoing transfer is uniformly distributed in the interval $[0, v]$ where $v$ is the total size of the ongoing transfer (which, in the stationary system, has distribution $V_{s}(\cdot)$, see Section IIA).

\section{A. Analysis of the Buffer Content Process}

Consider an AWP that starts transmission with an initial window of $w(0)$ packets. Let $w(n), n \geq 1$, denote the window size just after $\sum_{i=0}^{n-1} w(i)$ amount of data of the file is acknowledged.

Let the period where the $n^{\text {th }}$ window of the sequence $\{w(i), i \geq 0\}$ is getting served be called the $(n+1)^{t h}$ cycle. Thus $w(n-1)$ is the window at the start of the $n^{\text {th }}$ cycle and is also the amount of data served in $n^{t h}$ cycle. The $(n+1)^{t h}$ cycle starts when $w(n-1)$ amount of data is served after start of $n^{\text {th }}$ cycle. Let $W(n):=\sum_{i=0}^{n-1} w(i)$ denote the amount of data acknowledged till the start of $(n+1)^{t h}$ cycle (see Figure 4). Note that $W(0)=0$ by definition. The values of $w(n)$ and $W(n)$ for TCP's congestion avoidance and slow start phases are listed in Table I.

\begin{tabular}{|l|c|c|}
\hline Phase of TCP & $w(n)(\mathrm{pkt})$ & $W(n)(\mathrm{pkt})$ \\
\hline Congestion Avoidance & $n+1$ & $\frac{n(n+1)}{2}$ \\
Slow Start & $2^{n}$ & $2^{n}-1$ \\
\hline
\end{tabular}

TABLE I

TABLE SHOWING THE VALUES OF $w(n)$ AND $W(n)$ FOR TCP's CONGESTION AVOIDANCE AND SLOW START PHASES.

Note that the $w(n)^{\prime} s$ are not the only possible window sizes; they are the possible window sizes at the beginnings of cycles. For example, in slow start, the TCP window can take all integer values but $w(n)$ is restricted to integral powers of 2 .
Let $\gamma_{m}(z)$ denote the net input rate to the link buffer from an infinitely long session that has received $z$ amount of service in the $(m+1)^{t h}$ window. For slow start phase of TCP, $\gamma_{m}(z)=1$ because every $d z$ amount served brings in $2 d z$ and $d z$ amount leaves the link buffer. Note that the definition of $\gamma_{m}(z)$ does not depend on file size as it is defined for an infinitely long file.

Recall the function $U(v)$ defined in Section III. If the age $u$ of a file of size $v$ is such that $u>U(v)$ then the net data input rate into link buffer is -1 because no new data is sent for a unit amount of data served.

Note that if $v \in[W(m), W(m+1)]$, then $U(v) \in(W(m-$ 1), $W(m)]$.

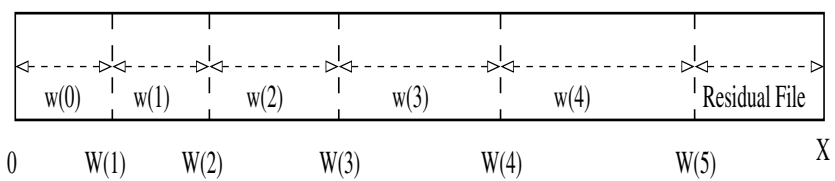

Fig. 4. The figure shows a file of size $X$ packets $(W(5)<X<W(6))$ split into the possible window sizes (at end of cycles) under a general AWP.

The idea now is to find the transform of distribution of windows which constitute the link buffer occupancy. Denote the LST of the window size distribution by $\tilde{g}(s)$. Once we have obtained $\tilde{g}_{s}(s)$ (the LST of spread distribution corresponding to $\tilde{g}(s)$ ), the distribution of the queue (link buffer occupancy random variable, denoted $Q$ ) is:

$$
\tilde{q}(s)=\sum_{k=0}^{\infty} \rho^{k}(1-\rho) \tilde{g}_{s}(s)^{k}=\frac{1-\rho}{1-\rho \tilde{g}_{s}(s)}
$$

Where we have used the fact that the probability of there being $n$ files active is $(1-\rho) \rho^{n}$ and as exactly one window worth of data from each file is in the buffer (due to zero propagation delay assumption), the probability of there being $n$ windows in the link buffer is also $(1-\rho) \rho^{n}$.

Thus we can obtain the mean buffer length and the variance of buffer occupancy once we have obtained $\tilde{g}_{s}(s)$. In particular, the mean buffer occupancy is given by:

$$
E Q=\left.\left(\frac{\rho}{1-\rho}\right)\left(-\frac{d}{d s} \tilde{g}_{s}(s)\right)\right|_{s=0}
$$

Observe that $E Q$ is finite iff $\left.\frac{d}{d s} \tilde{g}_{s}(s)\right|_{s=0}$ is finite.

Note that the stationary link buffer occupancy is actually a random sum of i.i.d. random variables distributed according to $\tilde{g}_{s}(s)$. Thus, using Proposition 2.9 of [22], it follows that if $\tilde{g}_{s}(s)$ corresponds to a sub-exponential distribution ([22]) then so does $\tilde{q}(s)$. In particular, if $\tilde{g}_{s}(s)$ is regularly varying with parameter $\beta$ than so is $\tilde{q}(s)$.

It can be easily shown, using the results for PS queue given in Section II-A, that

$$
\begin{aligned}
\tilde{g}_{s}(s)= & \sum_{n=0}^{\infty} \int_{v=W(n)}^{W(n+1)}\left[\int_{U(v)}^{v} e^{-s(v-u)} \frac{d u}{v}\right. \\
& +\int_{W(n-1)}^{U(v)} e^{-s\left(w(n-1)+\int_{0}^{u-W(n-1)} \gamma_{n-1}(z) d z\right)} \frac{d u}{v}
\end{aligned}
$$


$\left.+\sum_{m=0}^{n-2} \int_{u=W(m)}^{W(m+1)} e^{-s\left(w(m)+\int_{0}^{u-W(m)} \gamma_{m}(z) d z\right)} \frac{d u}{v}\right] d V_{s}(v)$

Where $n$ is used to condition on $v$ (the file size requirement) being in the $n^{t h}$ cycle, and $m$ is used to condition on $u$ (the age of the file) being in the $m^{t h}$ cycle. The integrand (with respect to $v$ ) above contains three terms to take care of the possibility where the age of file of is in the last window (i.e., $u>U(v)$ ) and hence whole of remaining file $v-u$ is in the link buffer.

The expected link buffer occupancy is, from Equations 9 and 10 and using $d V_{s}(v)=\frac{v d V(v)}{E V}$,

$$
\begin{aligned}
E Q= & \frac{\lambda}{(1-\rho)} \sum_{n=0}^{\infty} \int_{v=W(n)}^{W(n+1)}\left[\int_{U(v)}^{v}(v-u) d u\right. \\
& +\int_{u=W(n-1)}^{U(v)}\left(w(n-1)+\int_{0}^{u-W(n-1)} \gamma_{n-1}(z) d z\right) d u \\
+ & \left.\sum_{m=0}^{n-2} \int_{u=W(m)}^{W(m+1)}\left(w(m)+\int_{0}^{u-W(m)} \gamma_{m}(z) d z\right) d u\right] d V(v)
\end{aligned}
$$

Theorem 5.1: Under a general AWP with $\gamma_{m}(z) \geq$ $0, \quad \forall m, z$,

Proof: See Appendix IV.

$$
\begin{aligned}
& \frac{\lambda}{(1-\rho)} \sum_{n=0}^{\infty} w^{2}(n-1) V^{c}(W(n)) \leq E Q \\
& \quad \leq \frac{2 \lambda}{(1-\rho)} \sum_{n=0}^{\infty} w^{2}(n) V^{c}(W(n-1))
\end{aligned}
$$

Corollary 5.1: For congestion avoidance controlled transfer of Pareto distributed file sizes, $E Q$ is finite iff $\alpha>1.5$.

Proof: Follows from Theorem 5.1 with $V^{c}(v)=\frac{1}{v^{\alpha}}, w(n)=$ $n+1, W(n)=\frac{n(n+1)}{2}$.

Corollary 5.2: For slow start controlled transfer of Pareto distributed file sizes, $E Q$ is finite iff $\alpha>2$.

Proof: Follows from Theorem 5.1 with $V^{c}(v)=\frac{1}{v^{\alpha}}, w(n)=$ $2^{n}, W(n)=2^{n}-1$.

Above results clearly indicate that feedback control can result in lightening of the tail of link buffer occupancy when compared to an uncontrolled transfer of file. It can also be observed that an aggressive feedback control such as the slowstart phase of TCP may not result in lightening of buffer occupancy distribution; in this case the mean buffer occupancy is finite iff the second moment of the file size distribution is finite $^{3}$.

\footnotetext{
${ }^{3}$ In our recent research [17] we have obtained the exact tail behaviour of the buffer occupancy distribution. It is shown that for transfer of Pareto distributed files, (i) $Q^{c}(\cdot)$ is regularly varying with parameter $\alpha-1$ for TCP slow start controlled transfer, and (ii) $Q^{c}(\cdot)$ is regularly varying with parameter $2(\alpha-1)$ if the transfer are controlled using TCP congestion avoidance. The results of Corollaries 5.1 and 5.2 are thus reconfirmed.
}

Corollary 5.1 is interesting in view of the result of Section IV where it was shown that, for small arrival rates $\lambda$, the traffic into the link buffer $(a(t)$ process) is LRD for Pareto distributed file sizes with $1<\alpha<2$. Thus, we now have an example where the traffic into a queue is LRD but the mean queue length is finite; this is because the traffic into the queue is regulated using a closed loop control.

\section{CONCLUSION AND ONGOING WORK}

We have developed a framework for the analysis of processes related to the bottleneck link buffer under an adaptive window protocol (AWP) controlled transfer of randomly arriving finite volume files. The most important example of an AWP is TCP. It was shown that

1) When TCP's congestion avoidance or slow start algorithms are used to transfer Pareto distributed files, the traffic into the link buffer is LRD for small file transfer request arrival rates,

2) Under the congestion avoidance phase of TCP, the mean link buffer occupancy is finite when the Pareto distribution has shape parameter $\alpha>1.5$.

Thus we have given an example where, in the presence of LRD input to the link buffer, the buffer occupancy has finite mean. This suggests that the impact of long range dependence of Internet traffic may not be as severe as is usually predicted by means of an open loop analysis. This result also emphasises the importance of considering the interactions between the various processes as a result of closed loop congestion control.

Recent results from our ongoing research show that when the window is reduced in response to random marking, it results in further lightening of the tail behavior of buffer occupancy distribution while the input process still remains LRD. The work reported in this paper is for a zero propagation delay link; it will also be interesting to study how the results presented in this paper change as the propagation delay increases.

\section{APPENDIX I}

PROOF OF THEOREM 4.1

Let $V_{i}(t)$ and $Y_{i}(t), 1 \leq i \leq N(t)$, denote, respectively, the total and residual service requirements of the $i^{\text {th }}$ session active at time $t$. Now,

$$
\begin{aligned}
J_{1}(\tau) & =\operatorname{Ea}(0) a(\tau) I_{\left\{N(0)>0, K_{Y(0)}(\tau)=0\right\}} \\
& \geq \operatorname{Ea}(0) a(\tau) I_{\left\{N(0)=1, U\left(V_{1}(0)\right)-\left(V_{1}(0)-Y_{1}(0)\right)>\tau\right\}}
\end{aligned}
$$

which holds because when $N(0)=1, V_{1}(0)-Y_{1}(0)$ is the age of the file active at time 0 , and $U\left(V_{1}(0)\right)-\left(V_{1}(0)-Y_{1}(0)\right)>\tau$ implies that the source will still be sending data for this file until time $\tau$. Define the last term to be $J_{3}(\tau)$.

Note that $\left\{U\left(V_{1}(0)\right)-\left(V_{1}(0)-Y_{1}(0)\right)>\tau\right\} \subset\left\{V_{1}(0)>\right.$ $X(\tau)\}$, hence

$$
\begin{aligned}
& J_{3}(\tau)= \\
& E a(0) a(\tau) I_{\left\{N(0)=1, V_{1}(0)>X(\tau), Y_{1}(0)>V_{1}(0)-U\left(V_{1}(0)\right)+\tau\right\}}
\end{aligned}
$$


Plugging the distributions of $N(0), V_{1}(0)$ and $Y_{1}(0)$ using results given in Section II-A, we get from Equation 12,

$$
\begin{aligned}
J_{3}(\tau)=(1-\rho) \rho & \int_{v=X(\tau)}^{\infty} \int_{y=v-U(v)+\tau}^{v} a(0) \\
& E\left(a(\tau) \mid N(0)=1, V_{1}(0)=v, Y(0)=y\right) \frac{d y}{v} d V_{s}(v)
\end{aligned}
$$

At $\tau$ there could be other sessions active; these arrive in the interval $(0, \tau]$. Let us continue to use the index 1 at time $\tau$ for the session that was active at time 0 . Since $Y_{1}(\tau)>0$, (and noting that $V_{1}(\tau)=V_{1}(0)$ )

$$
a(\tau) \geq \frac{R_{V_{1}(\tau)}\left(V_{1}(\tau)-Y_{1}(\tau)\right)}{N(\tau)}=\frac{R_{V_{1}(0)}\left(V_{1}(\tau)-Y_{1}(\tau)\right)}{N^{\prime}(\tau)+1}
$$

where $N^{\prime}(\tau)$ is the number of sessions active at $\tau$ other than the tagged session which was active at time 0 . The inequality is obtained since there could be a positive rate from the other sessions at $\tau$.

By hypothesis, we have

$$
0<r \leq \inf _{0 \leq v<\infty} \inf _{0 \leq u<U(v)} R_{v}(u)
$$

Now, since $Y_{1}(\tau)>V_{1}(\tau)-U\left(V_{1}(\tau)\right)$, by definition of $r$ we have $R_{V_{1}(0)}\left(V_{1}(\tau)-Y_{1}(\tau)\right) \geq r$, hence,

$$
a(\tau) \geq \frac{r}{N^{\prime}(\tau)+1}
$$

so, for $y>\tau$,

$$
\begin{aligned}
& E\left(a(\tau) \mid N(0)=1, V_{1}(0)=v, Y(0)=y\right) \\
& \quad \geq r E\left[\frac{1}{N^{\prime}(\tau)+1} \mid N(0)=1, V_{1}(0)=v, Y(0)=y\right]
\end{aligned}
$$

also, since $V_{1}(0)-Y_{1}(0)<U\left(V_{1}(0)\right)$,

$$
a(0)=R_{V_{1}(0)}\left(V_{1}(0)-Y_{1}(0)\right) \geq r
$$

Hence, using above inequalities and that $d V_{s}(v)=\frac{v d V(v)}{E V}$,

$$
\begin{aligned}
J_{3}(\tau) \geq & (1-\rho) \lambda r^{2} \int_{v=X(\tau)}^{\infty} \int_{y=v-U(v)+\tau}^{v} \\
& E\left[\frac{1}{N^{\prime}(\tau)+1} \mid N(0)=1, V_{1}(0)=v, Y(0)=y\right] d y d V(v)
\end{aligned}
$$

Note that the conditions $N(0)=1, V_{1}(0)=v$ and $Y(0)=$ $y>\tau$ together imply that the file that was present at time 0 is also present at time $\tau$, and hence owing to the PS model $N^{\prime}(\tau)$ is independent of $v$ and $y$. Hence,

$$
\begin{array}{r}
J_{3}(\tau) \geq(1-\rho) \lambda r^{2} E\left[\frac{1}{N^{\prime}(\tau)+1} \mid N(0)=1, Y(0)>\tau\right] \\
\int_{v=X(\tau)}^{\infty} \int_{y=v-U(v)+\tau}^{v} d y d V(v)
\end{array}
$$

We know that (see Equation 3 in Section II-A with $k=1$ ),

$$
P\left\{N^{\prime}(\tau)=\left.n\right|_{N(0)=1, Y(0)>\tau}\right\} \sim_{\tau \rightarrow \infty}(1-\rho)^{2}(n+1) \rho^{n}
$$

Hence,

$$
E\left[\frac{1}{N^{\prime}(\tau)+1} \mid N(0)=1, Y(0)>\tau\right] \quad \sim_{\tau \rightarrow \infty} \quad(1-\rho)
$$

The proof follows with a change of variable (using $y=v-u$ ).

\section{APPENDIX II}

PROOF OF THEOREM 4.2

We condition as follows:

1) Condition on $N(0)$, the number of ongoing transfers at time 0 ,

2) given $N(0)$, condition on the total file size $V_{i}(0)$ of $i^{t h}$ ongoing transfer at time $0,1 \leq i \leq N(0)$,

3) given $N(0)$ and $V_{j}(0), 1 \leq j \leq N(0)$, condition on $Y_{i}(0)$, the residual size of the $i^{t h}, 1 \leq i \leq N(0)$ file having total size $V_{i}(0)$,

4) and, given $Y(0)=\sum_{i=1}^{N(0)} Y_{i}(0)$, the total residual file volumes at time 0 , condition on $\theta, 0<\theta<\tau$, the start of the first idle period after time 0

This yields, $J_{2}(\tau)=$

$$
\begin{aligned}
= & \sum_{n=1}^{\infty}(1-\rho) \rho^{n} \int_{v(n)=0}^{\infty} \int_{y(n)=0}^{v(n)} a(0) \int_{\theta=0}^{\tau} \\
& E\left(a(\tau) \mid N(\theta)=0, N\left(\theta^{-}\right)>0\right) b \sum_{i=1}^{n} y_{i}(\theta) d \theta \\
& \frac{d y(n)}{v(n)} d V_{s}(v(n))
\end{aligned}
$$

where $v(n)$ is a row vector of dimension $n$ with components $v_{1}, \ldots, v_{n}$ and $y(n)$ is a row vector of dimension $n$ with components $y_{1}, \ldots, y_{n}$. Also, abusing notation, $\frac{d y(n)}{v(n)}:=\frac{d y_{n}}{v_{n}} \ldots \frac{d y_{1}}{v_{1}}$ and $d V_{s}(v(n)):=d V_{s}\left(v_{n}\right) \ldots d V_{s}\left(v_{1}\right)$, and $b_{y}(\cdot)$ is the density of $B_{y}$.

Due to the Poisson arrival assumption

$$
E\left(a(\tau) \mid N(\theta)=0, N\left(\theta^{-}\right)>0\right)=E(a(\tau) \mid N(\theta)=0)
$$

is a function only of $\tau-\theta$.

Let $\Phi(\tau):=E(a(\tau) \mid N(0)=0)$ and denote by $\tilde{\Phi}(s)$ the Laplace Transform of $\Phi(\cdot)$. So,

$$
E\left(a(\tau) \mid N(\theta)=0, N\left(\theta^{-}\right)>0\right)=\Phi(\tau-\theta)
$$

Thus,

$$
\begin{gathered}
J_{2}(\tau)=\sum_{n=1}^{\infty}(1-\rho) \rho^{n} \int_{v(n)=0}^{\infty} \int_{y(n)=0}^{v(n)} a(0) \\
\int_{\theta=0}^{\tau} \Phi(\tau-\theta) b_{\sum_{i=1}^{n} y_{i}}(\theta) d \theta \frac{d y(n)}{v(n)} d V_{s}(v(n))
\end{gathered}
$$

Taking the Laplace Transform (LT) of Equation 13 and noting that

- $d V_{s}(v)=\frac{v d V(v)}{\sum^{n} E V}$

- $a(0)=\frac{\sum_{j=1}^{n} E V R_{v_{j}}\left(v_{j}-y_{j}\right)}{n}$

- the integral with respect to $\theta$ is a convolution of two terms which have LT given by $\tilde{\Phi}(s)$ and $\tilde{b}_{\sum_{i=1}^{n} y_{i}}(s)$, and,

- $\tilde{b}_{\sum_{i=1}^{n} y_{i}}(s)=\prod_{i=1}^{n} e^{-y_{i}(s+\lambda x(s))}$,

we get the following expression for the LT of $J_{2}(\tau)$.

$$
\begin{aligned}
& \tilde{J}_{2}(s)=\frac{s \tilde{\Phi}(s)}{s} \sum_{n=1}^{\infty}(1-\rho) \lambda^{n} \int_{v(n)=0}^{\infty} \int_{y(n)=0}^{v(n)} \\
& \frac{\sum_{j=1}^{n} R_{v_{j}}\left(v_{j}-y_{j}\right)}{n} \prod_{i=1}^{n} e^{-y_{i}(s+\lambda x(s))} d y(n) d V(v(n))
\end{aligned}
$$


It can be seen after some calculations that,

$$
\begin{aligned}
\tilde{J}_{2}(s)= & (1-\rho) \frac{s \tilde{\Phi}(s)}{s} \lambda\left[\int_{v=0}^{\infty} e^{-v(s+\lambda x(s))}\right. \\
& \left.\int_{u=0}^{v} e^{u(s+\lambda x(s))} R_{v}(u) d u d V(v)\right] \frac{s+\lambda x(s)}{s}
\end{aligned}
$$

We know that $\lim _{s \rightarrow 0} s \tilde{\Phi}(s)=\rho$. Let $s \tilde{\Phi}(s)=\rho+\kappa(s)$ where $\kappa(s) \rightarrow 0$ as $s \rightarrow 0$. Also, $\lim _{s \rightarrow 0} \int_{u=0}^{v} e^{u(s+\lambda x(s))} R_{v}(u) d u=$ $v$ so, $\int_{u=0}^{v} e^{u(s+\lambda x(s))} R_{v}(u) d u \stackrel{=}{=} v+\nabla(s) \quad$ where $\lim _{s \rightarrow 0} \nabla(s)=0$. Thus,

$$
\begin{aligned}
& \int_{v=0}^{\infty} e^{-v(s+\lambda x(s))} \int_{u=0}^{v} e^{u(s+\lambda x(s))} R_{v}(u) d u d V(v) \\
& \quad \sim_{s \rightarrow 0} \int_{v=0}^{\infty} e^{-v(s+\lambda x(s))} v d V(v)=-\left.\frac{d}{d z} \tilde{v}(z)\right|_{z=s+\lambda x(s)}
\end{aligned}
$$

Hence, for Pareto distribution, using algebra similar to that in [11], we get

$$
\begin{aligned}
\int_{v=0}^{\infty} e^{-v(s+\lambda x(s))} & \int_{u=0}^{v} e^{u(s+\lambda x(s))} R_{v}(u) d u d V(v) \\
& \sim_{s \rightarrow 0} \frac{\alpha}{\alpha-1}-\alpha^{2} \Gamma(-\alpha)(s+\lambda x(s))^{\alpha-1}
\end{aligned}
$$

Hence,

$$
\begin{aligned}
\tilde{J}_{2}(s) & \sim_{s \rightarrow 0}(1-\rho) s \tilde{\Phi}(s) \lambda \frac{s+\lambda x(s)}{s} \\
& \frac{\left[\frac{\alpha}{\alpha-1}-\alpha^{2} \Gamma(-\alpha)(s+\lambda x(s))^{\alpha-1}\right]}{s}
\end{aligned}
$$

The proof follows by noting that for $s \rightarrow 0, \quad x(s)=s E B+$ $o(s)=\frac{s E V}{1-\rho}+o(s)$, and also as $s \tilde{\Phi}(s) \sim_{s \rightarrow 0} \rho$, and that $E V=\frac{\alpha}{\alpha-1}$.

\section{APPENDIX III \\ A TAUberian THEOREM}

We reproduce an extension of a Tauberian Theorem of Widder [23] from [11] used in proof of Theorem 4.3.

Theorem 3.1: If

1) $\tilde{f}(s)=\int_{0}^{\infty} e^{-s t} f(t) d t, 0<s<\infty$

2) $f(t) \geq 0$ for $t \geq t_{0}$,

3) $\tilde{f}(s) \sim_{s \rightarrow 0^{+}} \frac{A}{s^{\beta}}$, for some $\beta>0$

then

$$
\int_{0}^{t} f(u) d u \sim_{t \rightarrow \infty} \frac{A t^{\beta}}{\Gamma(\beta+1)}
$$

\section{APPENDIX IV}

\section{PROOF OF THEOREM 5.1}

If $\gamma_{m}(z) \geq 0 \quad \forall m, z$ then the sequence $\{w(n), n \geq 0\}$ is nondecreasing and so, $\left(w(m)+\int_{0}^{u-W(m)} \gamma_{m}(z) d z\right) \leq w(m+$ 1) for $u \leq W(m+1)$. Thus we can upper bound the third term in the integrand (with respect to $v$ ) in the right hand side of Equation 11,

$$
\begin{array}{r}
\sum_{m=0}^{n-2} \int_{u=W(m)}^{W(m+1)}\left(w(m)+\int_{0}^{u-W(m)} \gamma_{m}(z) d z\right) d u \\
\leq \sum_{m=0}^{n-2} \int_{u=W(m)}^{W(m+1)} w(m+1) d u=\sum_{m=0}^{n-2} w(m) w(m+1) \\
\leq \sum_{m=0}^{n-2} w^{2}(m+1)
\end{array}
$$

Using the same argument, the second term in Equation 11 is

$$
\begin{aligned}
& \int_{u=W(n-1)}^{U(v)}\left(w(n-1)+\int_{0}^{u-W(n-1)} \gamma_{n-1}(z) d z\right) d u \\
& \leq \int_{u=W(n-1)}^{W(n)}\left(w(n-1)+\int_{0}^{u-W(n-1)} \gamma_{n-1}(z) d z\right) d u \leq w^{2}(n)
\end{aligned}
$$

Thus, using the above inequalities,

$$
\begin{array}{r}
\int_{v=W(n)}^{W(n+1)}\left[\int_{u=W(n-1)}^{U(v)}\left(w(n-1)+\int_{0}^{u-W(n-1)} \gamma_{n-1}(z) d z\right) d u\right. \\
\left.\quad+\sum_{m=0}^{n-2} \int_{u=W(m)}^{W(m+1)}\left(w(m)+\int_{0}^{u-W(m)} \gamma_{m}(z) d z\right) d u\right] d V(v) \\
\leq \int_{v=W(n)}^{W(n+1)} \sum_{m=0}^{n-1} w^{2}(m+1) d V(v)=w^{2}(n) V^{c}(W(n))
\end{array}
$$

where the last expression is obtained using some algebra. The first term in right hand side of Equation $11 \mathrm{can}$ be bounded from above as $v-u \leq w(n)$ for $v \in(W(n), W(n+1))$ and $U(v)<u<v$. Hence the first term is,

$$
\begin{aligned}
& \int_{v=W(n)}^{W(n+1)} \int_{U(v)}^{v}(v-u) d u d V(v) \\
& \quad \leq \int_{v=W(n-1)}^{\infty} w^{2}(n) d V(v)=w^{2}(n) V^{c}(W(n-1))
\end{aligned}
$$

Combining the upper bounds for the three terms of Equation 11 and noting that $V^{c}(W(n)) \leq V^{c}(W(n-1))$, we get

$$
E Q \leq \frac{2 \lambda}{(1-\rho)} \sum_{n=0}^{\infty} w^{2}(n) V^{c}(W(n-1))
$$

Now we can lower bound the three terms in right hand side of Equation 11. The first and second terms are clearly $\geq 0$. Also, if $\gamma_{m}(z) \geq 0$, then $\left(w(m)+\int_{0}^{u-W(m)} \gamma_{m}(z) d z\right) \geq$ $w(m)$. These observations along with some algebra gives the following lower bound

$$
E Q \geq \frac{\lambda}{(1-\rho)} \sum_{n=0}^{\infty} w^{2}(n-1) V^{c}(W(n))
$$

The result now follows from Equations 14 and 15.

\section{REFERENCES}

[1] Van Jacobson, "Congestion avoidance and control," in ACM SIGCOMM, 1988, pp. 314-329.

[2] Anurag Kumar, "Comparative Performance Analysis of Versions of TCP in Local Network with a Lossy Link," IEEE/ACM Transactions on Networking, vol. 6, no. 4, August 1998. 
[3] Jitendra Padhye, Victor Firoiu, Don Towsley and Jim Kurose, "Modeling TCP Throughput: A Simple Model and its Empirical Validation," in SIGCOMM'98, 1998.

[4] Arzad A. Kherani and Anurag Kumar, "Stochastic Models for Throughput Analysis of Randomly Arriving Elastic Flows in the Internet," in IEEE Infocom 2002, New York, June 2002.

[5] Mark E. Crovella and Azer Bestavros, "Self-Similarity in World Wide Web Traffic: Evidence and Possible Causes," IEEE/ACM Transactions on Networking, vol. 5, no. 6, pp. 835-846, December 1997.

[6] T.V. Lakshman and Upamanyu Madhow, "The performance of TCP/IP for networks with high bandwidth delay products and random loss," IEEE/ACM Transactions on Networking, vol. 5, no. 3, pp. 336-350, June 1997.

[7] H. Chaskar, T. V. Lakshman, U. Madhow, "TCP over wireless with link level error control: Analysis and design methodology," IEEE/ACM Transactions on Networking, vol. 5, no. 3, pp. 336-350, June 1999.

[8] M. Shreedhar and G. Varghese, "Efficient Fair Queuing using Deficit Round Robin," IEEE/ACM Transactions on Networking, 1996.

[9] S. Floyd and V. Paxson, "Difficulties in Simulating the Internet," IEEE/ACM Transactions on Networking, vol. 9, no. 4, pp. 392-403, 2001.

[10] D. Heath, S. Resnick and G. Samorodnitsky, "Heavy tails and long range dependence in on/off processes and associated fluid models," Mathematics of Operations Research, vol. 23, pp. 145-165, 1998.

[11] Anurag Kumar, K.V.S. Hari, R. Shobhanjali and S. Sharma, "Longrange dependence in the aggregate flow of TCP-controlled elastic sessions: An investigation via the processor-sharing model," in $\mathrm{Na}$ tional Conference on Communications, New Delhi, 2000, Available at http://ece.iisc.ernet.in/ anurag/pubm.html.

[12] W. E. Leland, M. S. Taqqu, W. Willinger and D. V. Wilson, "On the Self-Similar Nature of Ethernet Traffic (Extended Version)," IEEE/ACM Transactions on Networking, February 1994.

[13] B. Tsybakov and N. D. Georganas, "Self-Similar Traffic and Upper Bounds to Buffer-Overflow Probability in an ATM Queue," Performance Evaluation, 1998.

[14] I. Norros, "A Storage Model with Self-Similar Input," Queueing Systems, vol. 16, pp. 387-396, 1994.

[15] Y. Joo, V. Ribeiro, A. Feldmann, A. C. Gilbert and W. Willinger, "On the impact of variability on the buffer dynamics in ip networks," in 39th Annual Allerton Conf. on Communication, Control and Computing, 2001.

[16] A. Arvidsson, M. Roughan and T. Ryden, "On the Origins of LongRange Dependence in TCP Traffic," in ITC-17, 2001.

[17] Arzad A. Kherani and Anurag Kumar, "The Lightening Effect of Adaptive Window Control," To Appear in IEEE Communication Letters, Manuscript, available at http://ece.iisc.ernet.in $/$ anurag/pubm.html.

[18] J.W. Roberts and L. Massoulie, "Bandwidth Sharing: Objectives and Algorithms," in INFOCOM'99, 1999.

[19] Ronald W.Wolff, Stochastic Modelling and the Theory of Queues, Prentice Hall, Englewood Cliffs, 1989.

[20] F. P. Kelly, Reversibility and Stochastic Networks, John Wiley and Sons, 1979.

[21] Ward Whitt, "The M/G/1 Processor-Sharing Queue with Long and Short Jobs," Unpublished Report, available at: http://www.research.att.com/ wow/A12.html.

[22] K. Sigman, "A Primer on Heavy-Tailed Distributions," Queueing Systems, Theory and Applications, vol. 33, 1999.

[23] D.V. Widder, An Introduction to Transform Theory, Academic Press, New York, 1971. 